\title{
Gray literature in systematic reviews on population health in the Middle East and North Africa: protocol of an overview of systematic reviews and evidence mapping
}

Karima Chaabna ${ }^{1 *} \mathbb{D}$, Sohaila Cheema ${ }^{1}$, Amit Abraham ${ }^{1}$, Hekmat Alrouh ${ }^{1}$, Ravinder Mamtani ${ }^{1}$ and Javaid I. Sheikh ${ }^{2}$

\begin{abstract}
Background: Systematic review (SR) guidelines recommend extending literature search to gray literature in order to identify all available data related to the review topic. We aim to conduct an overview of SRs on population health in the Middle East and North Africa (MENA), to assess the methodology of these SRs, to produce an evidence map highlighting methodological gaps in SRs regarding gray literature searching, and to aid in developing future SRs by listing gray literature sources related to population health in MENA.

Methods/design: We will conduct an overview of SRs based on the Cochrane Handbook for Systematic Reviews of Interventions. This overview will be reported following PRISMA 2009 guidelines. Using comprehensive search criteria, we will search the PubMed database to identify relevant SRs published since 2008. Our primary outcomes are gray literature sources and study-level quality in the gray literature. We will include MENA countries with Arabic, English, French, and/or Urdu as primary official languages and/or media of instruction in universities. Two reviewers will independently conduct a multilevel screening on Rayyan software. Extraction of relevant data will be done on Statistical Package for the Social Sciences (SPSS) software. The methodological quality of included SRs will be assessed using the Assessment of Multiple Systematic Reviews (AMSTAR) tool. Any disagreements will be resolved by discussion and consensus.

We will estimate the overall proportion of SRs that used gray literature as one of their data sources. Subgroup analyses will be conducted to identify characteristics of these gray literature sources. Chi-squared and $t$ tests will be used to determine whether the differences between subgroups are statistically significant. Additionally, an evidence gap map will be constructed to highlight characteristics and quality of the gray literature used in SRs on population health in MENA and emphasize existing gaps in gray literature searching. We will also list gray literature sources identified in the included SRs stratified by country and research topic.
\end{abstract}

Discussion: This overview will comprehensively assess the overall quality of the SRs on population health issues in MENA. Our findings will contribute to the improvement of population health research practices in MENA.

Systematic review registration: The systematic review protocol was registered with the International Prospective Register of Systematic Reviews (PROSPERO) on 26 October 2018 (registration number CRD42017076736 (Syst Rev 2:4, 2013).

Keywords: Gray literature, Population health, Public health, MENA, North Africa, Middle East, Arab countries, Middle East and North Africa, Overview of systematic reviews

\footnotetext{
* Correspondence: kac2047@qatar-med.cornell.edu

'Institute for Population Health, Weill Cornell Medicine-Qatar, P.O. Box

24144, Doha, Qatar

Full list of author information is available at the end of the article
}

(c) The Author(s). 2018 Open Access This article is distributed under the terms of the Creative Commons Attribution 4.0 International License (http://creativecommons.org/licenses/by/4.0/), which permits unrestricted use, distribution, and reproduction in any medium, provided you give appropriate credit to the original author(s) and the source, provide a link to the Creative Commons license, and indicate if changes were made. The Creative Commons Public Domain Dedication waiver (http://creativecommons.org/publicdomain/zero/1.0/) applies to the data made available in this article, unless otherwise stated. 


\section{Background}

In healthcare, systematic reviews (SRs) and meta-analyses have become increasingly essential. Researchers, clinicians, and policymakers read them to keep up to date with their field and to make decisions [1, 2]. Thus, well-conducted SRs are important to implementing evidence-based medicine [3]. In 2008, the Institute of Medicine (IOM) recommended the development of methodological standards for SRs. Over the last decade, detailed materials guiding reviewers have been published; the original version of the Cochrane Handbook for Systematic Reviews of Interventions (version 5.0.1) was published in 2008 [4], the Preferred Reporting Items for Systematic Reviews and Meta-Analyses (PRISMA 2009) statement was published in 2009 [5], and IOM standards for SRs was published in 2011 [6]. These guidelines recommend extending literature searches to gray literature in order to identify all available data related to the review topic. The use of gray literature is important as it is likely to lead to a more complete view of available evidence [7]. Reviewers searching for gray literature on population health in the Middle East and North Africa (MENA) region might be challenged as the information may not be easy to search and retrieve because there were no central sources, which implies allocating considerable time and effort [7-9].

Non-English gray literature has been demonstrated to not affect results of reviews of intervention studies included in Cochrane Review Groups [10]. We will explore the use of gray literature by SRs of observational studies on population health in the MENA countries where English is not an official language. Our project objectives are to (i) conduct an overview of SRs (i.e., a systematic review of systematic reviews) on population health in MENA, (ii) assess the methodology of these SRs, (iii) produce an evidence gap map highlighting methodological gaps in SRs regarding gray literature searching, and (iv) aid in developing future SRs by listing gray literature sources related to population health in MENA.

\section{Methods}

Our protocol has been registered on PROSPERO (PROSPERO registration number CRD42017076736 [11]) and is reported following the items outlined in Preferred Reporting Items for Systematic Reviews and Meta-Analyses for Protocols (PRISMA-P 2015) [12]. Completed PRISMA-P 2015 checklist [13] can be found in Additional file 1.

\section{Research questions to be addressed}

The research questions we want to address are (i) what are the sources of gray literature used in the SRs on population health in MENA, (ii) what proportion of these SRs use gray literature, (iii) what are the characteristics of gray literature sources included in these SRs, (iv) are there differences between the SRs that use gray literature and those that do not, (v) are there differences between SRs using gray literature according to the type of gray literature, and (vi) what is the proportion of good quality studies from gray literature included in SRs on population health in MENA?

\section{Eligibility criteria}

Our study is an overview of published systematic reviews. As such, we will include only SRs [4]. Narrative reviews will be excluded. We will consider a publication as being an SR if it was stated that the publication is an SR and a systematic literature search of at least one electronic database was conducted and described in the method section along with a description of eligibility criteria and study selection. Reviews not reporting a systematic methodology will be excluded.

The following eligibility criteria are designed in compliance with the population-intervention-comparatoroutcome (PICO) model [14]. Populations in MENA are our populations of interest. We will use MENA region definition of The World Bank [15], World Health Organization-Eastern Mediterranean Region (WHO-EMR) [16], the Joint United Nations Programme on HIV/AIDS (UNAIDS) [17], and the Global Burden of Disease Study 2015 (GBD 2015) [18]. We are planning to extract data from the included SRs and from the primary studies identified by these SRs from gray literature sources. These SRs and primary studies are likely to be written in English or in the official languages or media of instruction of the selected countries. Hence, as our overview does not apply any language restriction in selecting the SRs and primary studies, we will include in our project those countries having Arabic, English, French, and/or Urdu as primary official languages and/ or media of instruction in universities. Arabic, English, French, and Urdu languages are the native languages of the reviewer team.

The 27 identified MENA countries [15-18], their primary official languages [19], media of instruction [20-47], and their selection status in our overview are listed in Table 1. We will include 20 countries, namely Algeria, Bahrain, Djibouti, Egypt, Iraq, Jordan, Kuwait, Lebanon, Libya, Morocco, Oman, Pakistan, Palestine, Qatar, Saudi Arabia, Sudan, Syria, Tunisia, United Arab Emirates, and Yemen. These selected MENA countries have a combined total population of over 560 million people, about $8 \%$ of the world's population [48].

We will include SRs on population health in MENA published since 2008 - the publication year of the first version of the Cochrane Handbook for Systematic Reviews of Interventions [4]. We will not restrict our overview to any health condition or intervention. Our primary outcomes 
Table 1 Countries of the Middle East and North Africa region included and excluded in the overview

\begin{tabular}{|c|c|c|c|c|c|c|c|}
\hline Country & The World Bank [15] & WHO-EMR [16] & UNAIDS [17] & GBD 2015 [18] & $\begin{array}{l}\text { Official primary } \\
\text { languages [19] }\end{array}$ & $\begin{array}{l}\text { Media of instruction in } \\
\text { universities and colleges }\end{array}$ & $\begin{array}{l}\text { Inclusion in } \\
\text { the overview }\end{array}$ \\
\hline Algeria & Yes & No & No & Yes & Arabic & French and Arabic [24] & Yes \\
\hline Bahrain & Yes & Yes & Yes & Yes & Arabic & Arabic and English [34] & Yes \\
\hline Djibouti & Yes & Yes & Yes & No & $\begin{array}{l}\text { Arabic and } \\
\text { French }\end{array}$ & French [47] & Yes \\
\hline Egypt & Yes & Yes & Yes & Yes & Arabic & Arabic and English [30] & Yes \\
\hline Iraq & Yes & Yes & Yes & Yes & Arabic & Arabic and English [29] & Yes \\
\hline Jordan & Yes & Yes & Yes & Yes & Arabic & Arabic and English [26] & Yes \\
\hline Kuwait & Yes & Yes & Yes & Yes & Arabic & Arabic and English [42] & Yes \\
\hline Lebanon & Yes & Yes & Yes & Yes & Arabic & $\begin{array}{l}\text { Arabic, French, and } \\
\text { English [31] }\end{array}$ & Yes \\
\hline Libya & Yes & Yes & Yes & Yes & Arabic & Arabic and English [25] & Yes \\
\hline Morocco & Yes & Yes & Yes & Yes & Arabic & Arabic and French [27] & Yes \\
\hline Oman & Yes & Yes & Yes & Yes & Arabic & Arabic and English [35] & Yes \\
\hline Pakistan & No & Yes & No & No & Urdu & English [39] & Yes \\
\hline Palestine & Yes & No & Yes & Yes & Arabic & English and Arabic [20] & Yes \\
\hline Qatar & Yes & Yes & Yes & Yes & Arabic & Arabic and English [43] & Yes \\
\hline Saudi Arabia & Yes & Yes & Yes & Yes & Arabic & Arabic and English [46] & Yes \\
\hline Sudan & No & Yes & Yes & Yes & Arabic & English [38] & Yes \\
\hline Syria & Yes & Yes & Yes & Yes & Arabic & $\begin{array}{l}\text { Arabic, French, and } \\
\text { English [28] }\end{array}$ & Yes \\
\hline Tunisia & Yes & Yes & Yes & Yes & Arabic & Arabic and French [45] & Yes \\
\hline United Arab Emirates & Yes & Yes & Yes & Yes & Arabic & English [22] & Yes \\
\hline Yemen & Yes & Yes & Yes & Yes & Arabic & $\begin{array}{l}\text { Arabic and } \\
\text { English }[39,44]\end{array}$ & Yes \\
\hline Afghanistan & No & Yes & No & Yes & Dari and Pashto & English and Arabic [21] & No \\
\hline Cyprus & No & Yes & No & No & Greek & $\begin{array}{l}\text { Greek, Turkish, and } \\
\text { English [33] }\end{array}$ & No \\
\hline Israel & Yes & No & No & No & Hebrew & Hebrew and English [41] & No \\
\hline Iran & Yes & Yes & Yes & Yes & Farsi & English and Farsi [23] & No \\
\hline Malta & Yes & No & No & No & Maltese & Maltese and English [32] & No \\
\hline Somalia & No & Yes & Yes & No & Somali & $\begin{array}{l}\text { Somali, Arabic, and } \\
\text { Italian [36] }\end{array}$ & No \\
\hline Turkey & No & No & No & Yes & Turkish & Turkish and English [40] & No \\
\hline
\end{tabular}

are gray literature sources in SRs on population health in MENA and study-level quality in the gray literature. In the current project, we will use the definition of gray literature provided at The Twelfth International Conference on Gray Literature in Prague in 2010 [8]. "Grey literature stands for manifold document types produced on all levels of government, academics, business and industry in print and electronic formats that are protected by intellectual property rights, of sufficient quality to be collected and preserved by libraries and institutional repositories, but not controlled by commercial publishers; i.e. where publishing is not the primary activity of the producing body.". Additionally, we will consider population health being defined as "the health outcomes of a group of individuals, including the distribution of such outcomes within the group" [49].

In order to provide an exhaustive list of gray literature sources, we will check the reference list of any review on available population health data sources in MENA identified during the multilevel screening process. If we identify relevant citations of gray literature sources related to population health in MENA, we will add them to our provided list of gray literature sources.

\section{Information sources and search strategy}

We will conduct a literature search (by AA) on PubMed [50] (Additional file 2). Key search terms will be related to countries' names, MENA populations' names, and 
MENA sub-regions' names such as North Africa, East Africa, and the Middle East. We will construct a broad search criteria using Boolean logic (OR and AND) to combine Medical Subject Headings (MeSH) terms and Title/Abstract words. We will use the MeSH Database [50] to search and identify relevant $\mathrm{MeSH}$ terms and use filters selecting reviews, SRs, and meta-analyses. The search will be limited to articles published since 2008 .

\section{Data management}

All reviews, SRs, and meta-analyses identified on PubMed will be imported into Endnote [51] and duplicates will be removed by HA; checking of this step will be conducted by KC. Two reviewers (AA and HA for publications in English, other combinations of reviewers for the remaining languages) will independently conduct a multilevel screening on Rayyan software, which was presented at the 22nd Cochrane Colloquium [52, 53]. In order to select potentially relevant SRs, we will first screen titles and abstracts of all unique reviews. We will then screen the included full texts to select the relevant SRs that will be included in our overview. Any disagreements during the screening process will be resolved by discussion and consensus between the reviewers. In the Title/Abstract screening step, we agreed that will be inclusive: if one of the reviewers thinks that the report needs to go through a full-text screening, we will include the report in this following step. For the full-text screening step, consensus is defined as $>50 \%$ of agreement between the authors. Reasons of exclusion in each screening steps will be recorded. One reviewer (AA for SRs and primary studies published in English, other reviewers according to the languages) will extract data on Statistical Package for the Social Sciences (SPSS), while a second reviewer (KC for SRs and primary studies published in English, other reviewers according to the languages) will check $100 \%$ of the extracted data. We will extract SRs' characteristics such as authors, country, corresponding author's institution, title, journal, year of publication, literature sources, SR period coverage, SR geographical coverage, and populations included. From the SRs, we will also extract characteristics of primary studies identified from gray literature (references, language and format of the study publication, risk of bias assessment, sampling methodology, health issue status, and response rate). Whenever one of primary studies' characteristics is not available in the corresponding SR, we will retrieve and extract data from the primary study report, if possible. A study report refers in previous reviews to a publication such as an article, a conference abstract, or a country-level report that presents study outcomes [54, 55]. Any disagreements during the extraction process will be resolved by discussion and consensus between the reviewers ( $>50 \%$ agreement between the reviewers).

\section{Assessment of methodological quality of included systematic reviews}

Using the Assessment of Multiple Systematic Reviews (AMSTAR) tool, we will assess the methodological quality of included SRs $[56,57]$.We will use AMSTAR tool rather than its revised version (R-AMSTAR), since R-AMSTAR is not yet validated [58] and since AMSTAR was recommended for assessing observational studies $[57,58]$. More specifically, we will appraise included SRs in our overview regarding the method used in literature searching, study selection, data collection, data analysis, publication bias assessment, and conflict of interest statement.

\section{Data synthesis and evidence mapping}

We will summarize SRs' methodology with a descriptive approach using tables presenting key characteristics of gray literature sources and quality of studies included from gray literature. From the SR-level quality assessment, we will estimate the overall proportion of SRs that used gray literature as one of their data sources. We will also provide a list of gray literature sources identified in the included SRs stratified by country and by research topic. This list format will be similar to the Canadian Agency for Drugs and Technologies in Health checklist "Grey Matters" in the field of drugs and technologies in health [59]. This Canadian list is used to ensure the retrieval of evidence-based agency reports, to help document the gray literature search process, and to conduct the gray literature search in a comprehensive way [59].

In the subgroup analysis, we will assess the differences between SRs that use gray literature and those that do not. We will estimate the proportion of SRs that used gray literature according to whether these SRs were produced by institutions located in MENA or outside MENA. We will compare language and format of gray literature sources included in SRs produced by institutions in MENA and among those produced by institutions outside MENA. Student's $t$ test and chi-squared test [60] will be used to determine whether the differences between subgroups are statistically significant ( $p$ value $<0.05)$. Bonferroni correction will be used to address the multiple testing issue regarding false positive.

Additionally, for each MENA country, we will review the five latest SRs containing gray literature searching. From the SRs, we will report on primary study-level quality assessment. We consider that SRs' authors are the experts in their research topics; as such, we will rely on their study-quality assessment. We are not aiming to compare the study-level quality between SRs but to estimate the overall proportion of good quality studies from the gray literature related to population health using the primary-study quality assessment. This proportion of good-quality primary studies included in our overview 
will be estimated after excluding duplicate studies using Endnote software [51]. When an SR does not provide quality assessment, we will assess study-level quality of included studies from the gray literature based on the Cochrane Handbook for Systematic Reviews of Interventions [4]. As described in previous reviews, we will classify studies as having a low, high, or unclear risk of bias in each of the three quality domains (sampling methodology, disease ascertainment, and response rate) [54, 55].

We will construct an evidence gap map defined as a visual depiction of the characteristics of evidence in a particular field [61] highlighting the type and quality of the gray literature used in published SRs. This evidence gap map will also emphasize existing gaps in gray literature searching [61]. This map will show the quality and the quantity of studies per language from gray literature included in SRs on population health in MENA. Our evidence gap map can aid in emphasizing gaps in SRs' literature searching and in planning future SRs on population health in MENA.

\section{Reporting of the findings}

Our overview will be reported following the preferred reporting items for overviews of systematic reviews [62] As overviews have a similar structure as systematic reviews, but include systematic reviews instead of primary studies [4], our overview's abstract will be reported following the PRISMA for Abstracts Checklist [63] and its methodological quality will be assessed using AMSTAR tool $[56,57]$. The findings will be disseminated via publication of a manuscript in a peer-reviewed journal and presented at relevant conferences.

\section{Discussion}

Our overview of SRs will enable us to identify, map, and compare the use of gray literature sources in published SRs on population health in MENA. The findings of this overview of SRs will have implications for research and evidence-based clinical and policy decisions. Our results will provide insight on available gray literature sources related to population health in MENA. The overview will describe to what extent researchers are missing reliable information when conducting SRs without including gray literature or when including a specific type of gray literature source. Our project will inform researchers, clinicians, and policymakers about the importance of including an extensive gray literature search in a region where official languages and/or media of instruction in universities include languages other than English.

\section{Ethics}

Ethical approval will not be needed, as this is a systematic review. Data used will not be individual patient data; therefore, there will be no concerns about privacy.

\section{Additional files}

Additional file 1: PRISMA-P* 2015 checklist: recommended items to address in a systematic review protocol [64]. (DOCX $27 \mathrm{~kb}$ )

Additional file 2: Search criteria. (DOCX $18 \mathrm{~kb}$ )

\section{Abbreviations}

AMSTAR: Assessment of Multiple Systematic Reviews; GBD 2015: Global Burden of Disease Study 2015; IOM: Institute of Medicine; MENA: Middle East and North Africa; MeSH: Medical Subject Headings; PICO: Populationintervention-comparator-outcome model; PRISMA 2009: Preferred Reporting Items for Systematic Reviews and Meta-Analyses; PRISMA-P 2015: Preferred Reporting Items for Systematic Reviews and Meta-Analyses Protocols; SPSS: Statistical Package for the Social Sciences; SR: Systematic review; UNAIDS: Joint United Nations Programme on HIV/AIDS; WHO-EMR: World Health Organization-Eastern Mediterranean Region

\section{Acknowledgements}

We would like to thank Dr. Ross MacDonald at Weill Cornell Medicine-Qatar for his technical assistance and insights as a librarian.

\section{Authors' contributions}

KC, SC, AA, HAR, RM, and JIS collectively contributed to the conception of the study. All authors were involved in the methodology development of the search strategy, the eligibility criteria, data extraction, and data analysis. The manuscript protocol was drafted by KC with support from SC, RM, and JIS. All authors read, edited, and approved the final manuscript.

Ethics approval and consent to participate

Not applicable.

Consent for publication

All authors approved the publication of the current protocol.

Competing interests

The authors declare that they have no competing interests.

\section{Publisher's Note}

Springer Nature remains neutral with regard to jurisdictional claims in published maps and institutional affiliations.

\section{Author details}

${ }^{1}$ Institute for Population Health, Weill Cornell Medicine-Qatar, P.O. Box 24144, Doha, Qatar. ${ }^{2}$ Office of the Dean, Weill Cornell Medicine-Qatar, Doha, Qatar.

Received: 25 September 2017 Accepted: 5 June 2018 Published online: 18 July 2018

\section{References}

1. Oxman AD, Cook DJ, Guyatt GH. Users' guides to the medical literature. VI. How to use an overview. Evidence-based medicine working group. Jama. 1994;272(17):1367-71.

2. Swingler $\mathrm{GH}$, Volmink J, loannidis JP. Number of published systematic reviews and global burden of disease: database analysis. Bmj. 2003;327(7423):1083-4.

3. Mulrow CD, Cook DJ, Davidoff F. Systematic reviews: critical links in the great chain of evidence. Ann Intern Med. 1997;126(5):389-91.

4. Higgins JPT, Green S (editors). Cochrane handbook for systematic reviews of interventions version 5.1.0 [updated March 2011]. The Cochrane Collaboration; 2011. Available from www.handbook.cochrane.org. Accessed 24 June 2018.

5. Liberati A, Altman DG, Tetzlaff J, Mulrow C, Gotzsche PC, loannidis JP, Clarke M, Devereaux PJ, Kleijnen J, Moher D. The PRISMA statement for reporting systematic reviews and meta-analyses of studies that evaluate health care interventions: explanation and elaboration. J Clin Epidemiol. 2009;62(10):e1-34.

6. Institute of Medicine (US) Committee on Standards for Systematic Reviews of Comparative Effectiveness Research: Finding what works in health care: standards for systematic reviews. Washington (DC): National Academies Press (US); 2011.

7. Mahood Q, Van Eerd D, Irvin E. Searching for grey literature for systematic reviews: challenges and benefits. Res Synth Methods. 2014;5(3):221-34. 
8. The Twelfth International Conference on Grey Literature [http://www. textrelease.com/gl12conference.html]. Accessed 24 June 2018.

9. Adams J, Hillier-Brown FC, Moore HJ, Lake AA, Araujo-Soares V, White M, Summerbell C. Searching and synthesising 'grey literature' and 'grey information' in public health: critical reflections on three case studies. Systematic reviews. 2016;5(1):164.

10. Hartling L, Featherstone R, Nuspl M, Shave K, Dryden DM, Vandermeer B. Grey literature in systematic reviews: a cross-sectional study of the contribution of non-English reports, unpublished studies and dissertations to the results of meta-analyses in child-relevant reviews. BMC Med Res Methodol. 2017;17(1):64.

11. Booth A, Clarke M, Dooley G, Ghersi D, Moher D, Petticrew M, Stewart L. PROSPERO at one year: an evaluation of its utility. Systematic reviews. 2013;2:4.

12. Moher D, Shamseer L, Clarke M, Ghersi D, Liberati A, Petticrew M, Shekelle P, Stewart LA, Group P-P. Preferred reporting items for systematic review and meta-analysis protocols (PRISMA-P) 2015 statement. Systematic reviews. 2015;4:1.

13. Shamseer L, Moher D, Clarke M, Ghersi D, Liberati A, Petticrew M, Shekelle P, Stewart LA, Group P-P. Preferred reporting items for systematic review and meta-analysis protocols (PRISMA-P) 2015: elaboration and explanation. Bmj. 2015;349:97647.

14. Richardson WS, Wilson MC, Nishikawa J, Hayward RS. The well-built clinical question: a key to evidence-based decisions. ACP J Club. 1995;123(3):A12-3.

15. Data: Wold Bank Country and Lending Groups [https://datahelpdesk.worldbank. org/knowledgebase/articles/906519-world-bank-country-and-lending-groups]. Accessed 24 June 2018.

16. Country groupings: Subregional country groupings for the global assessment of disease burden [http://www.who.int/quantifying_ehimpacts/ global/ebdcountgroup/en/]. Accessed 24 June 2018.

17. UNAIDS Middle East \& North Africa [http://www.unaidsmena.org/]. Accessed 24 June 2018.

18. GBD Compare | Viz Hub [http://vizhub.healthdata.org/gbd-compare/]. Accessed 24 June 2018.

19. The World Factbook [https://uww.cia.gov/library/publications/the-world-factbook] . Accessed 24 June 2018

20. Amara MH. Recent foreign language education policies in Palestine. Language Problems and Language Planning. 2003;27(3):217-32.

21. Baharustani R. Comprehensive study of higher education in Afghanistan Kabul: Afghanistan Investment Support Agency, Research and Planning Department; 2012

22. Belhiah H, Elhami M. English as a medium of instruction in the Gulf: when students and teachers speak. Lang Policy. 2015;14:3.

23. Ghorbani M, Zahed Alavi S. Feasibility of Adopting English-Medium Instruction at Iranian Universities. Current Issues in Education. 2014;17(1). Retrieved from https:/ cie.asu.edu/ojs/index.php/cieatasu/article/view/1277. Accessed 24 June 2018.

24. Nadia R. Teaching English in Algeria and educational reforms: an overview on the factors entailing students failure in learning foreign languages at university. Procedia-Social and Behavioral Sci. 2011;29:1327-33.

25. Tamtam A, Gallagher F, Naher S, Olabi A. The impact of language of instruction on quality of science and engineering education in Libya: qualitative study of faculty members. European Scientific J. 2013;9(31):19-36.

26. Abu-El-Haija A. Higher education in Jordan. In: Education, Audiovisual and Culture Executive Agency; ND. http://eacea.ec.europa.eu/tempus/ participating_countries/higher/jordan.pdf. Accessed 24 June 2018.

27. EP-Nuffic. Education system Morocco. In: 2nd edition edn: EP-Nuffic; 2015.

28. EP-Nuffic. Education system Syria. In: 1st edition edn: EP Nuffic; 2015.

29. EP-Nuffic. Education system Iraq. In: 2nd edition edn: EP-Nuffic; 2015.

30. EP-Nuffic. Education system Egypt. In: 2nd edition edn: EP-Nuffic; 2015.

31. EP-Nuffic. Education system Lebanon. In: 2nd edition edn: EP-Nuffic; 2016.

32. Council of Europe. Language Education Policy Profile: Malta. Council of Europe. 2015. http://www.coe.int/t/dg4/linguistic/Source/Profile-Malta_ final2015_EN.pdf. Accessed 24 June 2018.

33. Cd L'E. Language education policy profile: Cyprus. In: Conseil de L'Europe; N.D.

34. UNESCO International Bureau of Education. Bahrain. In: World Data on Education. 6th edition edn: UNESCO International Bureau of Education; 2006.

35. UNESCO International Bureau of Education. Oman. In: World Data on Education. UNESCO International Bureau of Education; 2011.

36. United States Agency for International Development. Country Context Report - Somalia. In: USAID; N.D. http://pdf.usaid.gov/pdf_docs/Pnadg222. pdf. Accessed 24 June 2018.

37. British University of Yemen [https://bu-yenet/]. Accessed 24 June 2018.

38. Education-Sudan [http://countrystudies.us/sudan/51.htm]. Accessed 24 June 2018.
39. English as a medium of instruction - a growing global phenomenon [https://www.britishcouncil.es/sites/default/files/british_council_english_as_ a_medium_of_instruction.pdf]. Accessed 24 June 2018.

40. Medium of instruction [http://ww4.ticaret.edu.tr/internationaloffice/mediumof-instruction/]. Accessed 24 June 2018.

41. Academic year and language of instruction in Israel Education System [http://www.israeleducation.info/Education-System/academic-year-andlanguage-of-instruction-in-israel-education-system.html]. Accessed 24 June 2018.

42. Language of instruction in Kuwait [http://www.kuwaiteducation.info/ Education-System/Language-of-instruction.html]. Accessed 24 June 2018.

43. Qatar University admissions [http://www.qu.edu.qa/students/admission/ graduate/faq.php]. Accessed 24 June 2018.

44. Sana'a University [http://www.su.edu.ye//. Accessed 24 June 2018.

45. Education in the Maghreb: Tunisia [http://wenr.wes.org/2006/04/wenr-apr2006-education-in-tunisia]. Accessed 24 June 2018.

46. Higher education in Saudi Arabia [http://wenr.wes.org/2014/11/highereducation-in-saudi-arabia]. Accessed 24 June 2018.

47. Universite de Djibouti [http://www.univ.edu.dj/]. Accessed 24 June 2018.

48. Total population - both sexes. De facto population in a country, area or region as of 1 July of the year indicated. Figures are presented in thousands. World Population Prospects: The 2015 Revision [https://esa.un. org/unpd/wpp/Download/Standard/Population/]. Accessed 24 June 2018.

49. Kindig D, Stoddart G. What is population health? Am J Public Health. 2003; 93(3):380-3.

50. PubMed.gov US National Library of Medcine [http://www.ncbi.nlm.nih.gov/ pubmed]. Accessed 24 June 2018.

51. EndNote X7 [http://endnote.com/product-details]. Accessed 24 June 2018.

52. Ouzzani M, Hammady H, Fedorowicz Z, Elmagarmid A. Rayyan — a web and mobile app for systematic reviews. Systematic Reviews. 2016;5(1):210.

53. Elmagarmid A, Fedorowicz Z, Hammady H, llyas I, Khabsa M, Ouzzani M. Rayyan: a systematic reviews web app for exploring and filtering searches for eligible studies for Cochrane Reviews. In: Evidence-Informed Public Health: Opportunities and Challenges Abstracts of the 22nd Cochrane Colloquium: 2014. Hyderabad: Wiley; 2014.

54. Chaabna K, Kouyoumjian SP, Abu-Raddad L. Hepatitis C virus epidemiology in Djibouti, Somalia, Sudan, and Yemen: systematic review and metaanalysis. PLoS One. 2016;11(2):e0149966.

55. Chaabna K, Mohamoud YA, Chemaitelly H, Mumtaz GR, Abu-Raddad LJ. Protocol for a systematic review and meta-analysis of hepatitis C virus (HCV) prevalence and incidence in the Horn of Africa sub-region of the Middle East and North Africa. Systematic reviews. 2014;3:146.

56. Shea BJ, Grimshaw JM, Wells GA, Boers M, Andersson N, Hamel C, Porter AC, Tugwell P, Moher D, Bouter LM. Development of AMSTAR: a measurement tool to assess the methodological quality of systematic reviews. BMC Med Res Methodol. 2007;7:10.

57. Pieper D, Mathes T, Eikermann M. Can AMSTAR also be applied to systematic reviews of non-randomized studies? BMC Res Notes. 2014;7:609.

58. Pieper D, Buechter RB, Li L, Prediger B, Eikermann M. Systematic review found AMSTAR, but not R(evised)-AMSTAR, to have good measurement properties. J Clin Epidemiol. 2015;68(5):574-83.

59. Grey Matters: a practical tool for searching health-related grey literature [https://www.cadth.ca/resources/finding-evidence/grey-matters]. Accessed 24 June 2018

60. Pearson $\mathrm{K}$. On the criterion that a given system of deviations from the probable in the case of a correlated system of variables is such that it can be reasonably supposed to have arisen from random sampling. Philosophical Magazine Series. 1900;5(50):157-75.

61. Miake-Lye IM, Hempel S, Shanman R, Shekelle PG. What is an evidence map? A systematic review of published evidence maps and their definitions, methods, and products. Systematic reviews. 2016;5:28.

62. Bougioukas Kl, Liakos A, Tsapas A, Ntzani E, Haidich AB. Preferred reporting items for overviews of systematic reviews including harms checklist: a pilot tool to be used for balanced reporting of benefits and harms. J Clin Epidemiol. 2018;93:9-24.

63. Beller EM, Glasziou PP, Altman DG, Hopewell S, Bastian H, Chalmers I, Gotzsche PC, Lasserson T, Tovey D. Group PfA: PRISMA for Abstracts: reporting systematic reviews in journal and conference abstracts. PLoS Med. 2013;10(4):e1001419.

64. GL'99 Conference Program. Fourth International Conference on Grey Literature: New Frontiers in Grey Literature. In: 1999. Washington D.C. USA; 1999. 